\title{
STABILITY IMPROVEMENT OF GRID CONNECTED PV SYSTEM USING BATTERY
}

\author{
Sanju Hussain ${ }^{1}$, Devanand $\mathbf{C} \mathbf{N}^{2}$ \\ ${ }^{1}$ MTech Student, Energy Management, Govt. Model Engineering College, Kerala, India \\ ${ }^{2}$ Assistant Professor, Mechanical Engineering, Govt. Model Engineering College, Kerala, India
}

\begin{abstract}
The battery storage and time based tariff are not introduced in our country yet. This paper proposes a new multi stage converter topology intended for single phase grid connected PV system with battery storage. Also focuses on incorporating optimized energy storage to minimize cost of investment and losses. The system works on different modes of operation depending on solar, load, grid and battery profiles. The voltage control is implemented using load angle control of inverter current. In order to eliminate the grid connected current distortion caused by phase error; the phase compensation and PR controller strategy are employed in current control loop to improve the traditional proportional integration controller. The simulation results shows that the output current zero crossing distortion is eliminated and the power factor has improved by the performance of load angle control.
\end{abstract}

Keywords: Stability, Battery Optimization, Power Factor.

\section{INTRODUCTION}

Solar photovoltaic power generation is gaining more attention with possible energy crisis and environmental issues. Also the need for pollution free green energy has created a keen interest towards photovoltaic power generation. Integration of PV power generation systems to the grid plays an important role in securing the electric power supply in an environmentally-friendly manner. Grid-connected PV inverters are needed to extract the energy from the PV modules and feed it into the utility grid while ensuring the power quality follows certain grid interconnection standards such as IEEE-1547. The output power generated from the solar panels is intermittent in nature and varies with the irradiance level. Hence to make the system more reliable, a battery is included in the system which helps in mitigation of voltage fluctuations, reduction of transient harmonics, improves quality of power, stabilizes grid by drawing less power from grid. Power converters play a key part as the interface between grid and energy sources. In practice, considering the electrical security of PV panels and the common mode current issues, grid-connected $\mathrm{PV}$ inverters are required to be electrical isolated. Many studies could be found in the literature on grid connected PV system, grid stability and inclusion of energy storage with PV systems in both national and international levels. John K. Pedersen et. al. 2005 have detailed various systems of single phase inverters for small scale distribution generators. For acceptation and handling of wide variations on the input voltage, multi stage inverters are suggested. Yuanbin He et. al. 2011 discussed that for single phase inverters, comparing conventional LCL filter LLCL power filters are more advantageous. Jaume Miret et. al. 2009 used selective resonant harmonic compensators for the mitigation of high frequency harmonics. Bin Yu et. al 2006 explained a predictive control mechanism to reduce the harmonic distortion in inverter output current. Gitizadeh et.al.2014 presented sizing of energy storage of grid connected PV system using general algebraic modeling system to minimize cost of battery. Wang et. al.2009 presented a topology to reduce ripples with reduced size dc link.Here a multi stage PV inverter integrated that includes proper controllers with phase compensation and optimized energy storage are proposed. Importance of proper scheduling dispatch based on state of charge of storage and other constraints are discussed. Also the power factor improvement by load angle control without active power curtailment is proposed.

\section{THE PROPOSED MULTISTAGE INVERTER}

A $2 \mathrm{KW}$ single phase grid connected PV inverter is designed. For the proper power conditioning basic components such as solar PV array, DC-DC converter, DC-AC inverter, battery and controllers are arranged suitably. Fig.1 shows the proposed multistage converter topology with high frequency transformer isolation. 


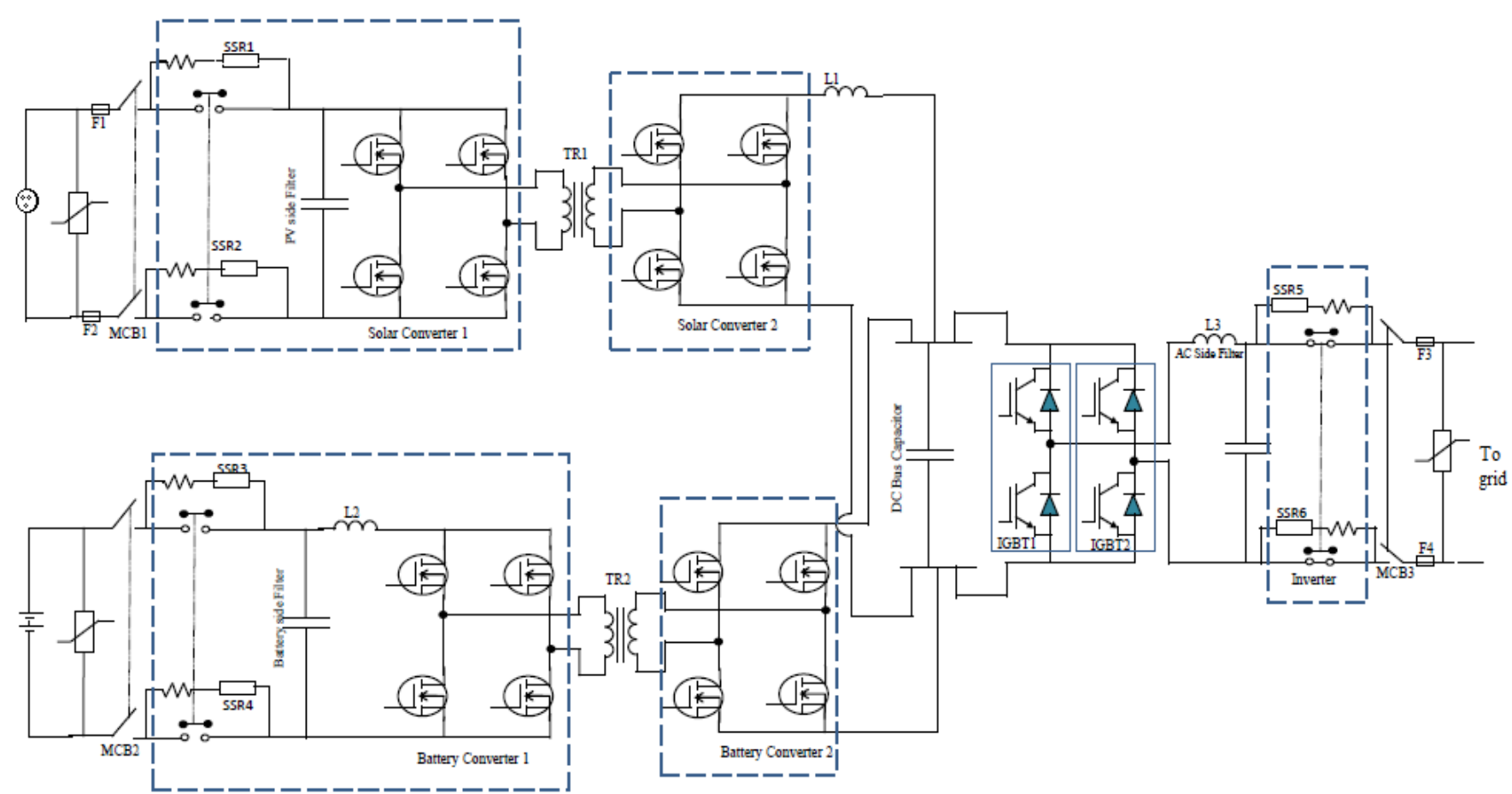

Fig 1: Circuit diagram of the proposed system

The DC-DC conversion part can be realized in two parts. The first unidirectional DC-DC converter is connected with the solar panel and the second bidirectional DC-DC converter connected with the battery, since it has to work in the battery charging and discharging modes. The full bridge converter topology is used for solar PV converter and battery converter, with high frequency isolation transformer connected to $400 \mathrm{~V}$ DC bus instead of traditional boost converters to reduce ripples thereby improvement in power quality. The electrical isolation in the high-frequency link is essential because it permits easy array grounding, flexibility in selecting array voltage range, array isolation from the utility in case of fault, and safety of personnel. It also help in reducing the size and volume of the system. The design of decoupling capacitor is important. That is if a $48 \mathrm{~V} 300 \mathrm{w}$ rating and the ripple voltage is $3 \mathrm{~V}$,the decoupling capacitor value is $7 \mathrm{mF}$ to get a PV utilization factor of $98 \%$. A single phase full bridge inverter is used for converting solar or battery voltage to $230 \mathrm{Vac}$. The inverter works in grid connected mode of operation, switching at line frequency. The inverter is realized using IGBTs and a LC filter to get a sinusoidal output. By using high frequency transformer we can create a high voltage on secondary side.To derive the equation of ripple voltage [Billy et.al. 2005], we have to consider input power $P_{\text {in }}$ from $P V$ and inverter output power $\mathrm{P}_{\text {out. }}$

Mathematically,

$$
\Delta \vartheta_{d c}=\frac{P_{m}}{4 \omega C_{d c} V_{d c}}
$$

where $\Delta \mathrm{v}_{\mathrm{dc}}$ is the ripple voltage, $\omega$ is the angular frequency of the grid, $\mathrm{P}_{\mathrm{m}}$ is the peak instantaneous grid power, the capacitor $\mathrm{C}_{\mathrm{dc}}$ acts as a buffer for absorbing the difference between $\mathrm{P}_{\text {in }}$ and $\mathrm{P}_{\text {out }}$ and $\mathrm{V}_{\mathrm{dc}}$ is the dc link voltage respectively. From the expression it is clear that the ripple voltage reduces when the dc link voltage increases. Therefore to connect the dc link capacitance on the high voltage bus is more beneficial. From Fig 1. the solar converter1 controls MPPT and also helps for voltage amplification. The solar converter2 controls the output current. Battery converter1 controls the charge-discharge current and is bidirectional and battery converter2 helps to generate the required dc link voltage that also is bidirectional.

\section{OPERATIONAL MODES OF THE SYSTEM}

There are different modes of operation controlled by the inverter according to the estimated data of PV power, state of charge of the battery, load demand, grid voltage existence and peak load shaving. In mode I, when the PV power is clearly available that is during sunny days, the inverter is connected to grid. The solar converter (SC) will performs the maximum power point tracking function and the current at maximum power point is calculated and the DC link voltage is regulated to $400 \mathrm{~V}$ at the input of inverter.

Mode $I-P V \rightarrow S C \rightarrow$ Inverter $\rightarrow$ Grid

When the PV power generation exceeds the low load profile, also when the grid voltage fluctuates within allowable standards, it will move to mode II, where the excess power is stored in the battery and also when state of charge is below $50 \%$, the battery will get charged from the PV.

Mode II - Excess power $\rightarrow$ Battery converter $\rightarrow$ Battery

hen grid voltage fluctuation occurs during peak hours and 
also when the PV power is not available or if the ramping rate is very high and the state of charge is above $50 \%$ the battery will supplies power to local priority loads or to grid during peak hours as in mode III. When the power generated stored in battery and supplies to the local load, it will act as as a standalone mode even when the grid voltage is not available.

\section{Mode III- Battery $\rightarrow$ Battery converter $\rightarrow$ Inverter $\rightarrow$ Grid/local loads}

When battery is in critical condition, that is if the state of charge is below $20 \%$, and if PV is not available, it will move to mode IV.

Mode IV - Grid $\rightarrow$ Inverter $\rightarrow$ Battery converter $\rightarrow$ Battery

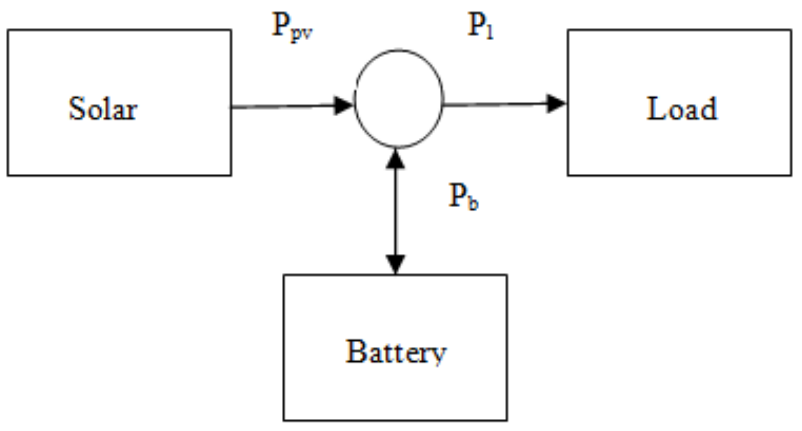

Fig 2: Block diagram of power flow

\section{OPTIMIZATION OF BATTERY}

Many literatures could be found on the economic and technical overview of battery storage systems for large scale electricity storage. It is necessary for a battery optimization, to minimize the cost of investment and losses. After optimization, a simple energy management strategy is also included to avoid over charging or discharging of battery. In this paper, we are demonstrating how a PV battery dynamics incorporated in to an optimization formulation. The goal is to determine the optimal power distribution between the PV array and battery to meet the load demand. Here, we consider a simple power flow model, which can provide a first order approximation to the PV system energy management optimization. Ultimately, this formulation results in a linear program.

A forecasted load profile of critical load of $300 \mathrm{~W}$ and the total load varying from $300 \mathrm{~W}$ to $1100 \mathrm{~W}$ during early day hours, remaining steady during noon at $600 \mathrm{~W}$ and increasing towards $1500 \mathrm{~W}$ in the evening hours was used. The power demand on all the time steps $\mathrm{k}=0, \ldots \ldots \ldots \ldots \ldots, \ldots \mathrm{N}$ is estimated. Consider the block diagram in Fig.2. which demonstrates the power flow in the PV system.

The solar panel produces power $\mathrm{P}_{\mathrm{pv}}$, the battery can store or release power $\mathrm{P}_{\mathrm{b}}$ and both must provide the power demand $\mathrm{P}_{1}$. Therefore we have the power conservation equality constraint

$$
P_{p v}(k)+P_{b}(k)=P_{l}(k), \forall k=0, \ldots N
$$

where positive value for $\mathrm{P}_{\mathrm{b}}$ is taking correspond to discharging power. The battery has energy storage dynamics given by state variable $\mathrm{E}(\mathrm{k})$, which indicates the current energy (in KJ or KHz)stored in the battery. This can be expressed as

$$
\begin{gathered}
E(k+1)=E(k)-P_{b}(k) \Delta t, \forall k=0 . . N-1 \\
E(0)=E_{0}
\end{gathered}
$$

where $\Delta \mathrm{t}$ is the sampled time step and $\mathrm{E}_{0}$ is the initial battery energy level. We have to ensure net zero energy transfer for the battery and it is assumed that the final capacity of the battery is $E(0)$ for making it ready for the next schedule.

$$
E(N)=E(0)
$$

In reality, this will never be realized with equality exactly, so we relax this constraint in to the following inequality.

$$
0.95 E(0) \leq E(N) \leq 1.05 E(0)
$$

In addition we also assume the energy level $\mathrm{E}(\mathrm{k})$ and battery power $\mathrm{P}_{\mathrm{b}}(\mathrm{k})$ are limited to the inequality constraints.

$$
\begin{aligned}
E^{\min } & \leq E(k) \leq E^{\max } \\
P_{b}^{\min } & \leq P_{b}(k) \leq P_{b}^{\max }
\end{aligned}
$$

Collecting all the above mentioned equation produces an optimization program and the total charge discharge energy of the battery over the entire horizon can be calculated as

$$
J=\sum_{k=0}^{N-1} P_{b}(k) \Delta t
$$

$\mathrm{J}$ represents the total charging or discharging power over the entire horizon. By minimizing the value of $\mathbf{J}$ helps us to calculate the optimized size of the battery storage.

\subsection{Linear Programming Formulation}

We can obtain a linear program (LP) of the form as expressed in equation 1o using equalities and inequalities taken from the equations 3-8. storage.

$$
\begin{array}{r}
\min x \quad f^{T} x \\
\text { subject to } \quad A x \leq b, A_{c q} x=b_{c q}
\end{array}
$$

where the decision variable given by

$$
x=\left|P_{0}(0), P_{0}(0), \ldots . . P_{0}(N-1), E(0), E(1), \ldots E(N-1), E(N)\right|^{T}
$$

and we have $2 \mathrm{~N}+1$ decision variables. The above equations are properly arranged to formulate $\mathrm{f}, \mathrm{A}, \mathrm{b}, \mathrm{A}_{\mathrm{eq}}$ and $\mathrm{b}_{\mathrm{eq}}$. We can 
see that

$$
f=[\Delta t, \ldots . \Delta t, 0, \ldots . .0]^{T}
$$

Then we rewrite the the equality and the inequality constraints in a matrix form using $\mathrm{x}$ defined in equation 11 as

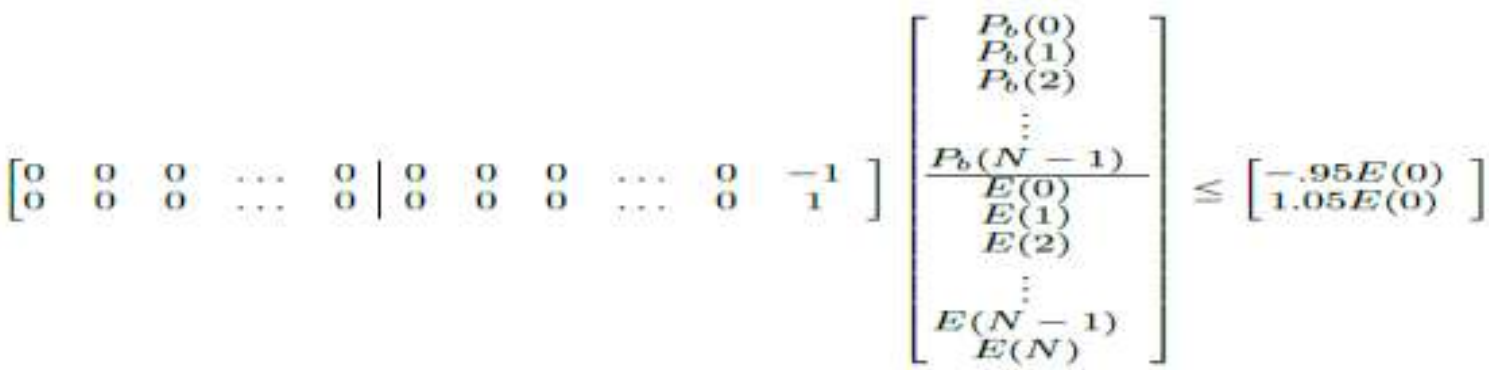

$L b=\left[\begin{array}{lllllllllll}-P_{\min } & -P_{\min } & -P_{\min } & \ldots & -P_{\min } & E_{\min } & E_{\min } & E_{\min } & \ldots & E_{\min } & E_{\min }\end{array}\right]$
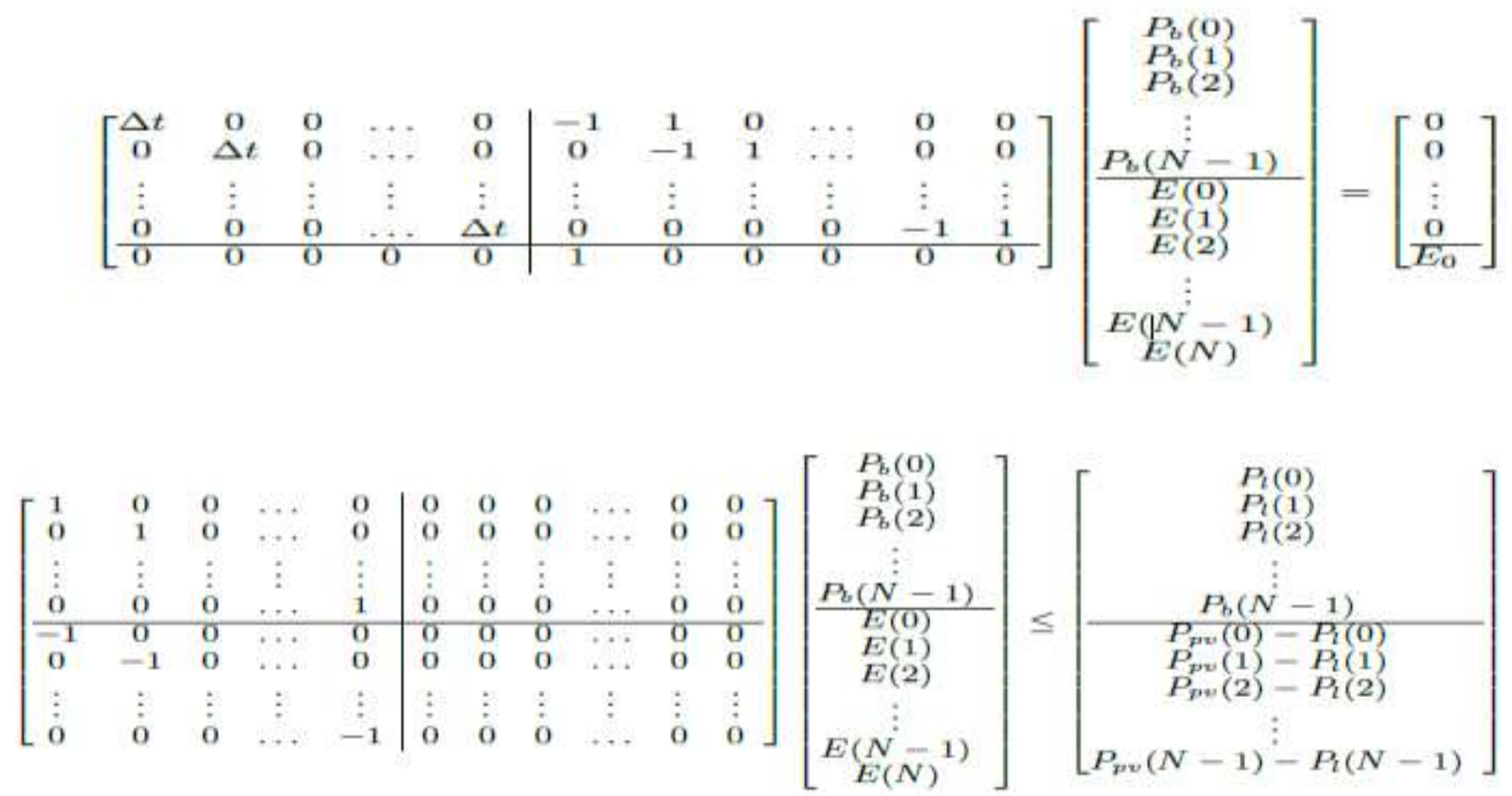

where $\mathrm{Lb}$ and $\mathrm{Ub}$ are lower and upper bounds of the power and energy that is charged or discharged. $\mathrm{P}_{\mathrm{pv}}(\mathrm{k})$ and $\mathrm{P}_{\mathrm{l}}(\mathrm{k})$ is the power generated by the PV and the load demand at the time step k.Concatenating the matrices, we obtain A and b.Now,the energy management problem has encoded in to the matrices $\mathrm{f}, \mathrm{A}, \mathrm{b}, \mathrm{A}_{\mathrm{eq}}, \mathrm{b}_{\mathrm{eq}}$ and can be solved using linear program solving.

\section{CONTROL ALGORITHMS}

The DC/AC/AC inverters remove the intermediate DC link to reduce volume and improve efficiency with cyclo converter as output stage, but the control system become more complicated with increased number of power switches. The
DC/AC/DC/AC inverters simplify the control system with decoupling capacitor in the intermediate DC link. The solar converter current controlling is done using the control scheme described in the following Fig.3.The solar converter 1 that follows MPPT controller, after finding maximum power point, new PV reference voltage is compared with the sensed voltage from PV and calculate the orthogonal current to establish new reference voltage from MPPT. Then the reference current is 


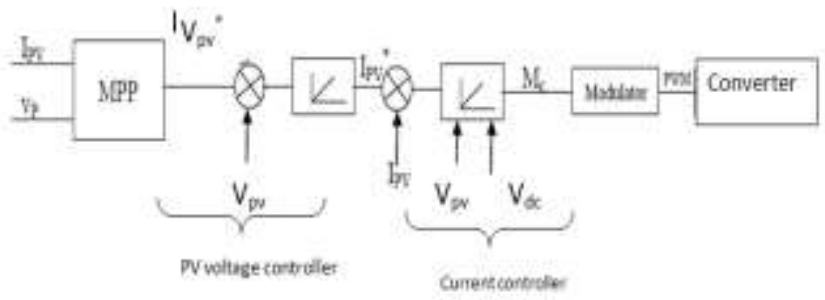

Fig 3: Converter controller

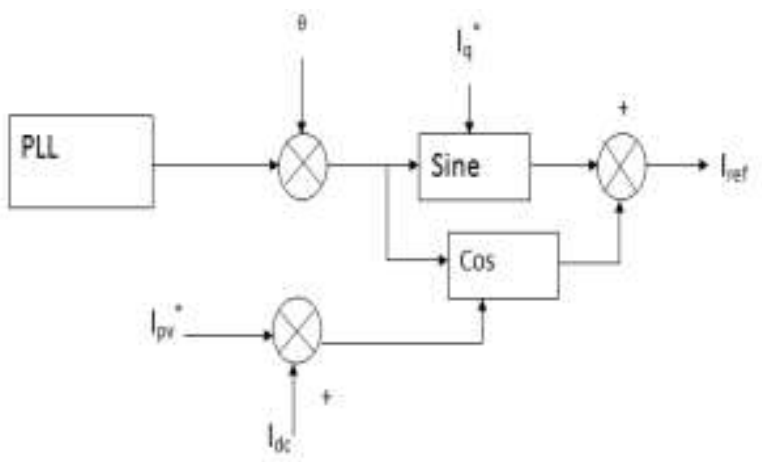

Fig 4: Phase compensation scheme

compared with the sensed PV current and modulation index by the current mode controller is calculated to generate the PWM signal for the switching of the converter. The phase error will lead to current distortion when output stage inverter switches at the zero crossing point and disrupt the system stability. For the above consideration, the phase compensation is employed to improve the inner control loop to solve the problem of grid connected current distortion caused by static phase error. With the phase angle accessed by PLL (phase-locked loop) from the grid voltage, phase compensation is done to make the output current synchronous to grid. The phase compensation scheme is shown in Fig.4.

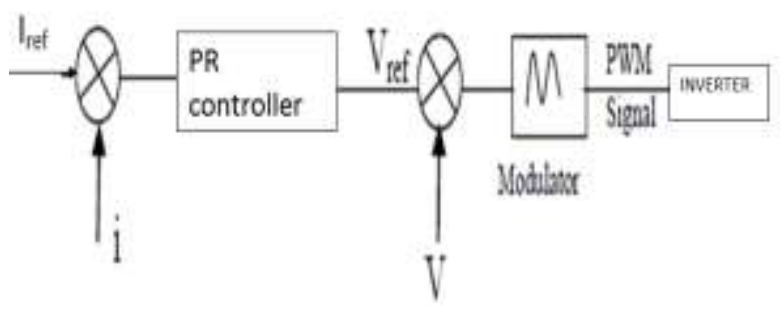

Fig 5: Inverter control

The controller for the inverter helps to maintain the DC link voltage at $400 \mathrm{Vdc}$. A PI controller is used to generate the error signal which is proportional to the active current for controlling the dc link voltage. The PI controller is.

$$
H(s)_{P I}=K_{P}+\frac{K_{I}}{S}
$$

The controller for inverter in the grid connection mode is shown in the Fig.5.The traditional PI controller for inverter cannot achieve zero steady state error and it has poor distur- bance rejection capability while tracking the sinusoidal signals, hence PR controller along with the phase compensation is used here. The sensed PV current to be injected to grid is compared with active current required to maintain the dc link voltage, after synchronizing with phase and frequency of the grid, is compared with the output inverter current. The reactive component that is generated by the load angle ad justment in the system is also considered here. The reactive component helps to adjust the fluctuation of grid voltage. The reference current generated is now compared with the output inverter current and pass through the PR controller. The PR controller act as a harmonic compensation tuned at line frequency and the reference voltage thus generated which is proportional to

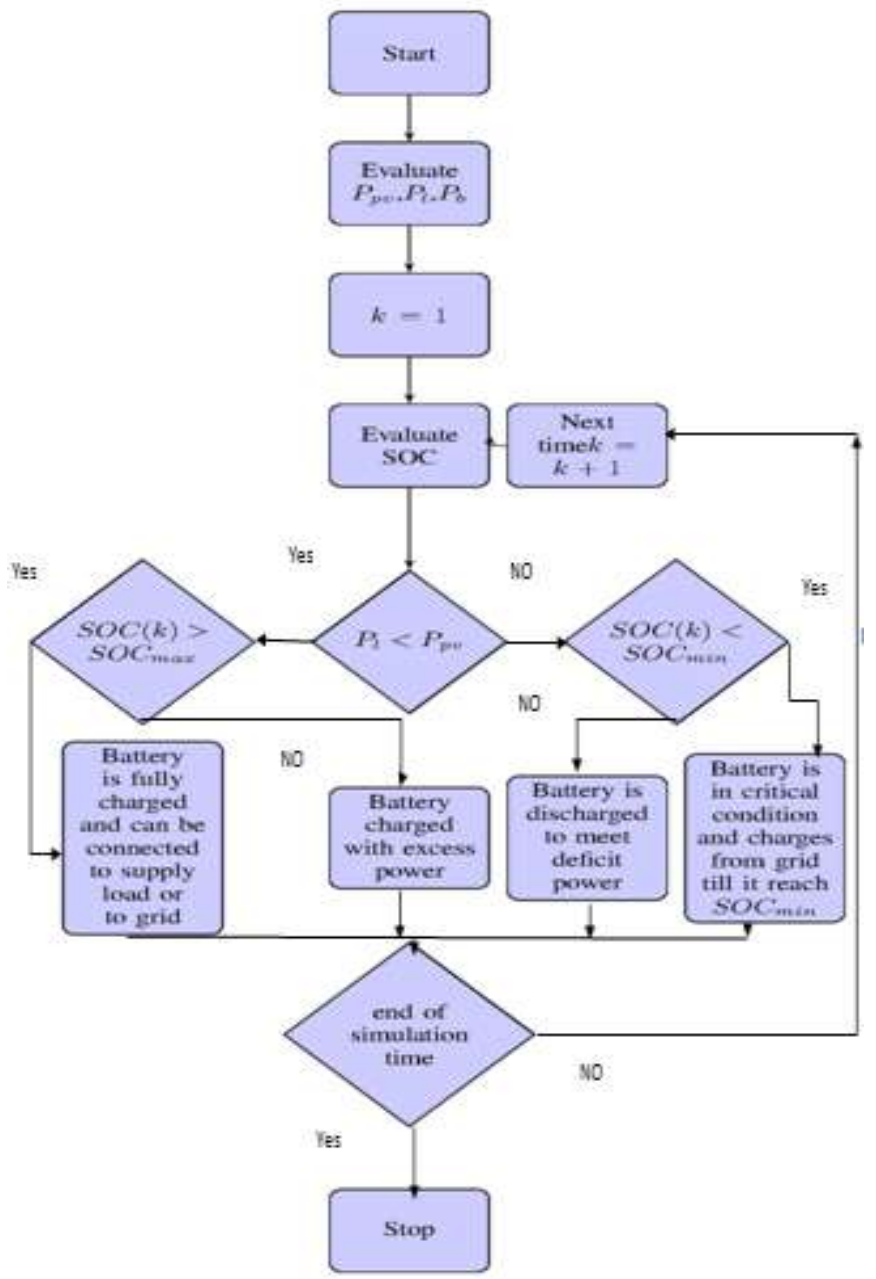

Fig 6: Energy management flow chart

the required inverter current, is compared with the grid voltage and calculates the modulation index to generate the pulses for switching the inverter at line frequency.

The PR controller is

$$
H(s)_{P I}=K_{P}+\frac{K_{I} \times 2 \omega_{c} S}{S^{2}+2 \omega_{c} S+\omega_{0}^{2}}
$$

where $\omega_{\mathrm{c}}$ and $\omega_{0}$ are bandwidth and resonant frequency. 
The battery operates in charge or discharge mode based on the power surplus or deficit occurring in the system. The energy management strategy is depicted in the flow chart of Figure 6.

At the instant of discharging, we can adjust the power injected to the grid according to equation 15.

$$
P_{\text {tot }}=P_{l}-\eta_{\text {inv }} P_{p v}-\left(D R \times V_{b} \times D O D\right) \eta_{b}
$$

where $\mathrm{P}_{\text {tot }}$ is the power injected to the grid, $\mathrm{P}_{1}$ load demand, $\mathrm{DR}$ is the discharging rate, $\mathrm{V}_{\mathrm{b}}$ is the battery voltage, DOD is the depth of discharge. From equation it is clear that by adjusting DR or the discharging current of the battery, the power injected to the grid can be controlled. Similarly during charging mode of battery,

$$
P_{\text {tot }}=\eta_{\text {inv }}\left(P_{p v}-\left(C R \times V_{b}\right)-P_{l}\right.
$$

where $\mathrm{CR}$ is the charging rate of battery current. The power taken from the grid can be controlled by adjusting the charging current of the battery. For voltage rise mitigation, active power curtailment and reactive power control methods are usually used. For voltage rise mitigation [K M Muttaqi et.al 2014], using a moving average method or the involvement of battery during voltage ramping has been suggested. Also, the authors reduced the reverse power flow by injecting less power to the grid during voltage rising time. In this paper to control voltage rising, load angle control method is proposed. By avoiding active power curtailment, the reactive power to and from the grid is controlled using energy storage. As depicted in Fig.7 (a) during voltage rising situation, the load angle is controlled and shifted such that inverter current is adjusted, to act as sink of reactive power, load angles the power from IR to I1'R, that is from quadrant I to IV.When voltage dip occurs due to overload, the load angle shift in-order to reduce that lag angle, at that time the PV generator shifts from reactive power sink to reactive power source. At quadrant III, the PV generator sinks both active and reactive power, at that time it act as a load, so the operating point should shift from quadrant III to IV, the battery discharges and when the operation is in quadrant I, the excess power generated is charged by the battery and shift to quadrant II as depicted in Fig.7(b).

$\varphi$ is the power angle between the grid voltage and the inverter output current. And $\delta$ is the load angle between the grid voltage and the inverter output voltage. The phase diagram is shown in Fig. 8. The following equations can be represented:

$$
\begin{aligned}
& E=U+j X_{s} I \\
& E \sin (\delta)=X_{s} I \cos (\psi)
\end{aligned}
$$

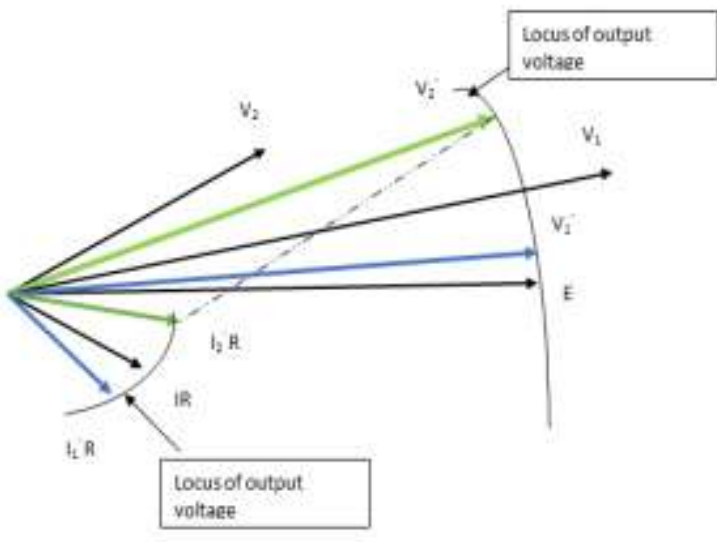

(a) Phasor diagram

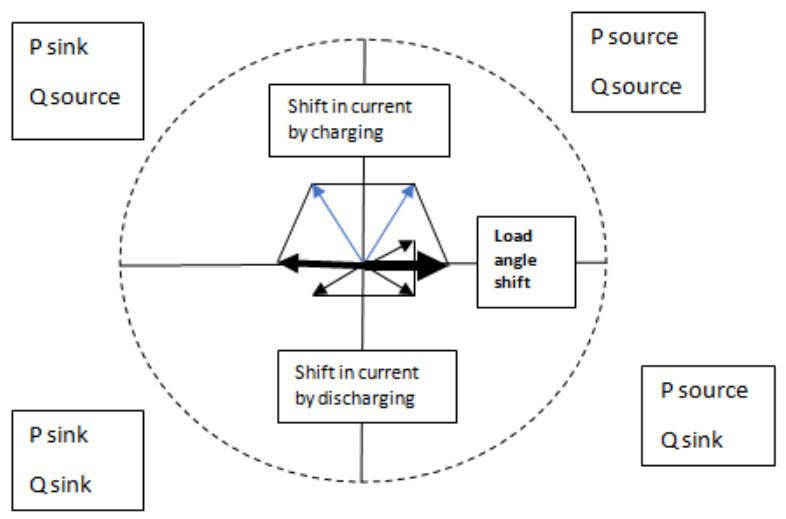

(b) Four quadrant operation

Fig 7: Phasor diagram and four quadrant operation of load angle control

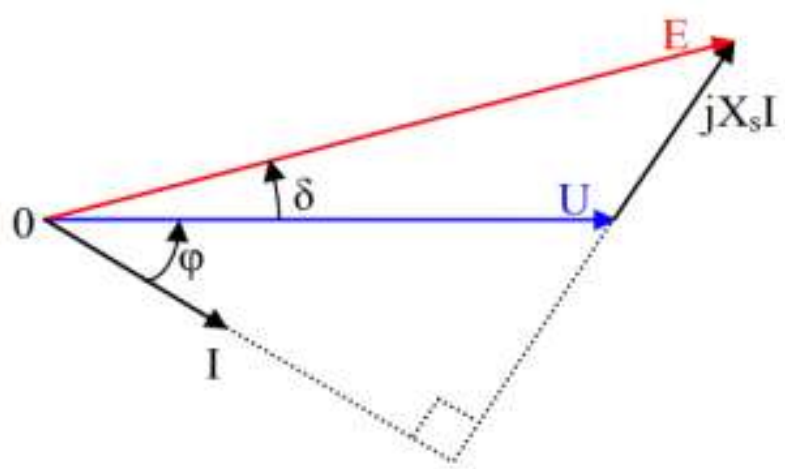

Fig 8: Phase diagram with grid voltage and load angle

The active power $\mathrm{P}$ to the grid from inverter

$$
P=U I \cos (\psi)=\frac{U E}{X_{\AA}} \sin (\delta)
$$

And the reactive power $\mathrm{Q}$ to the grid from inverter

$$
Q=\frac{U E}{X_{u}} \cos (\delta)-\frac{U^{2}}{X_{t}}=\frac{U}{X_{g}}(E \cos (\delta)-U)
$$


According to figure 8, equations (19) and (20), the power flow can be adjusted by controlling the inverter output voltage (E) and load angle $\delta$.From equation (19) and (20), the active and reactive power depend on both the inverter output voltage magnitude $\mathrm{E}$ and the load angle $\delta$. Xs is the filter reactance. So, the active power injected into the grid can be controlled by the phase difference between grid voltage and inverter output voltage. At the same time, the reactive power can be controlled by the inverter output voltage magnitude $\mathrm{E}$. The change in load angle is reflected in the reference current as it is derived from the voltage of inverter.

\section{RESULTS AND DISCUSSION}

For analysis, the rated capacity of grid connected inverter is selected as $2 \mathrm{KW}, 230 \mathrm{Vac}, 50 \mathrm{~Hz}$.For the battery optimisation, a set of residential load variation Fig.9(a) and PV data Fig.9(b) from Trivandrum is estimated for an interval of 0.6 minutes for 24 hours. Therefore, for a complete horizon, $\mathrm{N}=2400$ time steps and $\Delta \mathrm{t}=36$ s. Therefore we have 4801 decision variables for our linear program(LP).During the starting state of discharge, the energy of the battery is taken as $50 \%$ of the assumed capacity. Here, for a $2 \mathrm{KW}$ solar array and $1.5 \mathrm{~kW}$ load (maximum value in the load profile), the assumed battery capacity is $1.5 \mathrm{~kW} . \mathrm{E}(0)=\mathrm{E}_{0}=2700 \mathrm{~kJ}$, Emin = $1620 \mathrm{~kJ}, \mathrm{E}_{\max }=3780 \mathrm{~kJ}, \mathrm{P}_{\mathrm{bmin}}=-1.5 \mathrm{~kW}, \mathrm{P}_{\mathrm{b} \max }=1.5 \mathrm{~kW}$ (maximum load) for a $2 \mathrm{~kW}$ system. From the iteration using LP, we obtain $\mathrm{P}_{\text {batt }}$ (discharge) $=103.4 \mathrm{Ah}$ at $48 \mathrm{~V}, \mathrm{P}_{\text {batt }}$ (charge $)=106 \mathrm{Ah}$ at $48 \mathrm{~V}$.The total optimized battery power is calculated as Fig.9(c). The battery capacity is optimized as 48v 110 Ah at $\mathrm{C} / 20$ discharge rate.

A simulation model is built in MATLAB/Simulink based on the above analysis to check the inverter controls. The PR controller was designed with bandwidth of .5rad/s, $\mathrm{K}_{\mathrm{p}}=5.1322, \mathrm{~K}_{\mathrm{R}}=2073.000$ and PI controller uses a

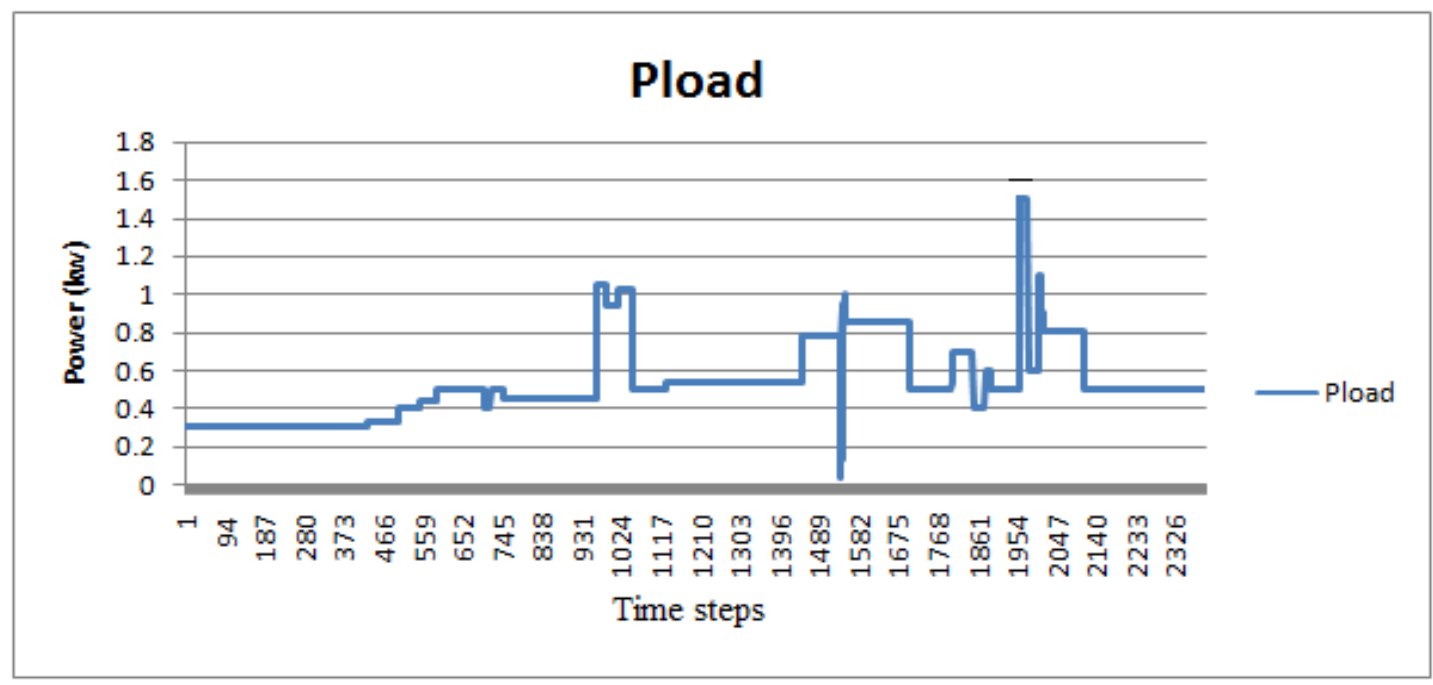

(a) Load variation

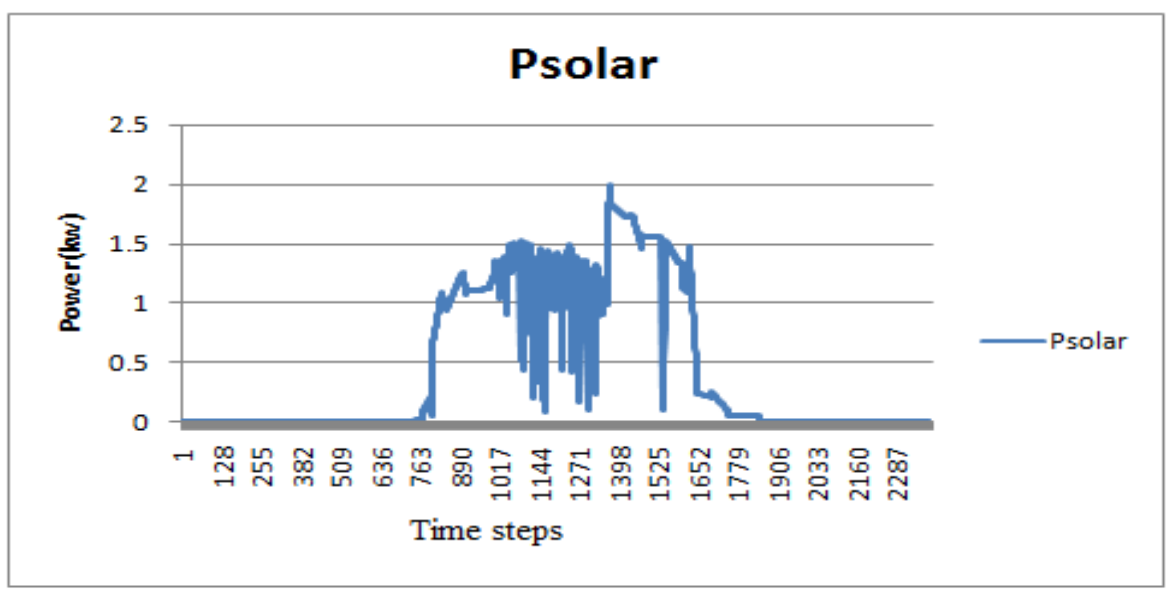

(b) Generated Solar power 


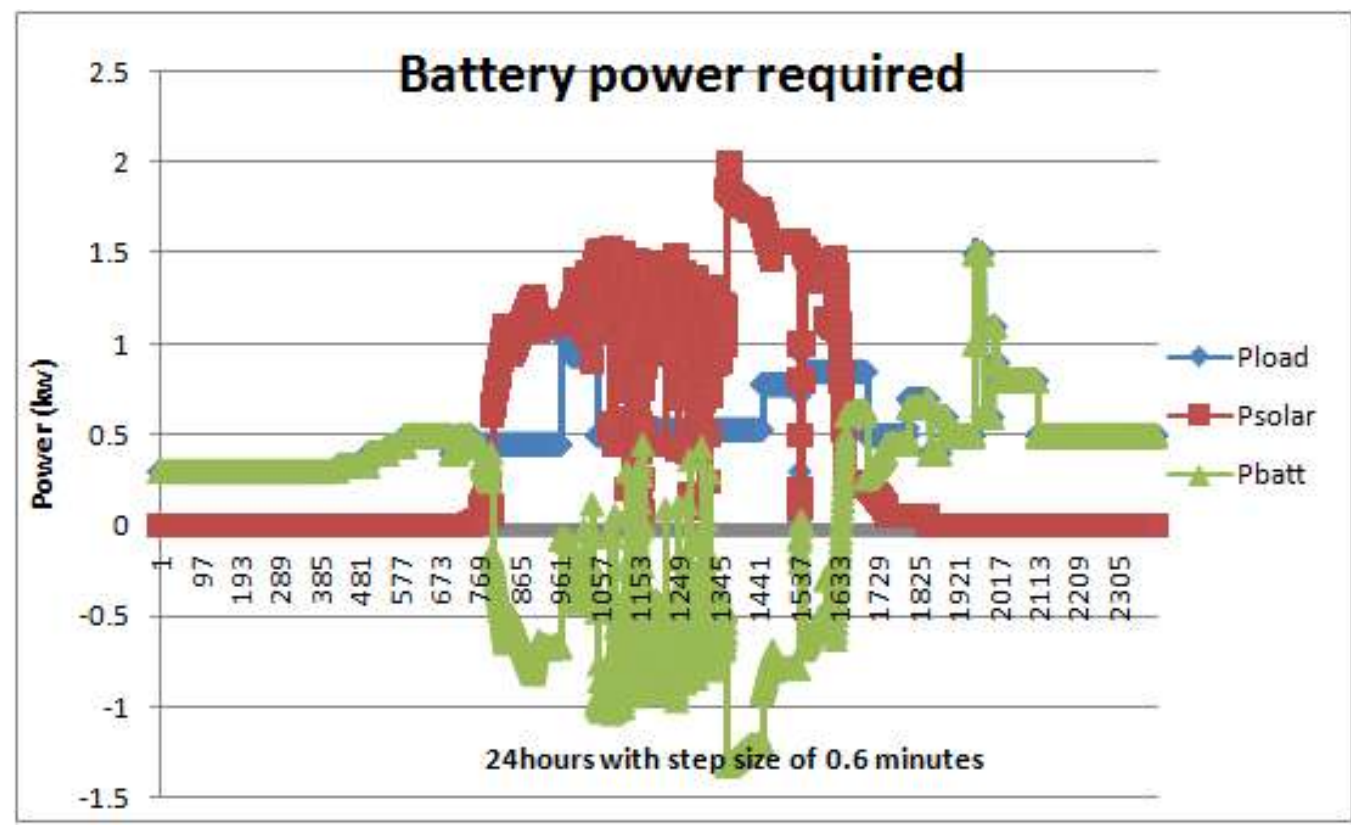

(c) Optimized size of battery

Fig 9: Battery size optimization

damping factor of 0.8 with natural frequency of $10 \mathrm{KHz}, \mathrm{K}_{\mathrm{P}}=$ $4.331, \mathrm{~K}_{\mathrm{I}}=2169$. During stability enhancing inverter controllers are not used, by using traditional controller, as predicted the output current is almost synchronous to grid. However, it is also seen that there is current distortion at zero crossing and harmonics are present which are serious issues. Simulation results without using stability enhancing controllers, are shown in Fig.10.By using inverter controllers which are discussed above for stability enhancement, the inverter can zero-voltage and zero-current switches without current distortion. Thus the harmonics are reduced, along with the usage of properly designed filters. The simulation results are shown in Fig.11.For understanding the performance of the system, with and without the stability enhancing system using energy storage which are discussed, simulation is done by varying grid voltage and solar irradiance.Fig. 12 will depicts the operation of the system. A reduction of irradiance is applied at $1 \mathrm{~s}$ which lasts for $1 \mathrm{~s}$, and a voltage reduction or sag is applied at $2.5 \mathrm{~s}$ lasts for $1 \mathrm{~s}$. A decrease in active power occurs in both cases without controllers and with controlling system.With the decrease of irradiance leads to leading reactive power without the controlling systems and reduction of reactive power with controlling systems which depicts the help of reactive power compensation with the controlling system. When a decrease of voltage applied results in decrease of reactive power without the stability enhancing controls and an increase of reactive power with the stability enhancing controls. Thus we can see power factor is improved.

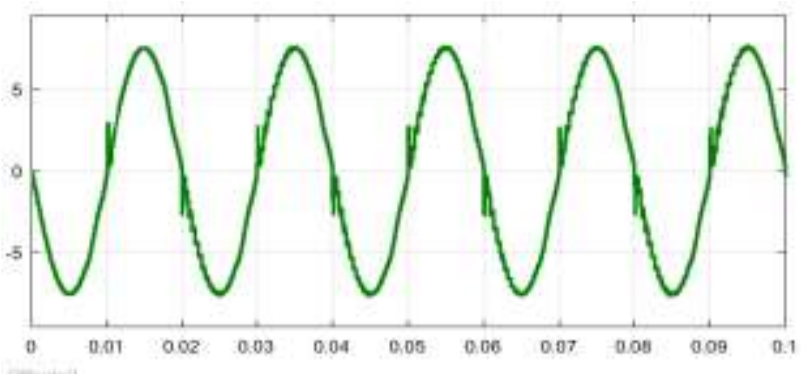

(a). Grid current.

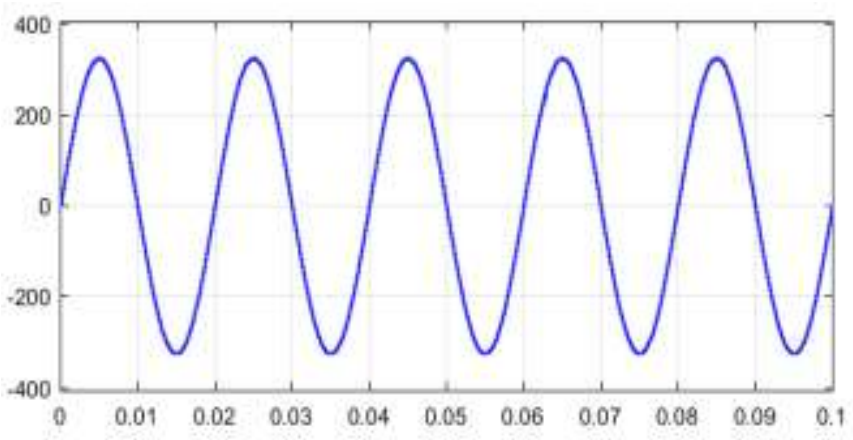

(b) Grid voltage

Fig 10: Grid voltage and current without using stability enhancing controllers 


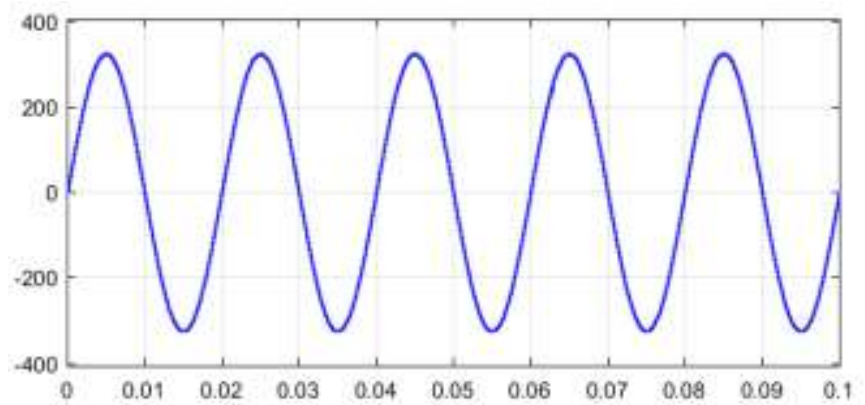

(a) Grid voltage

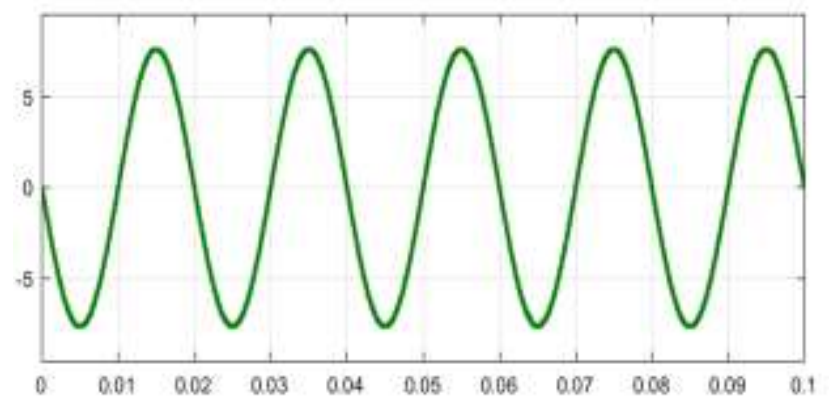

(b) Grid current

Fig 11: Grid voltage and current using stability enhancing controllers

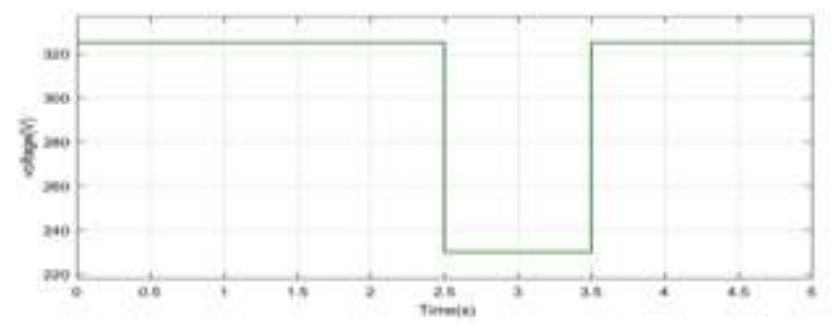

(a). Grid voltage

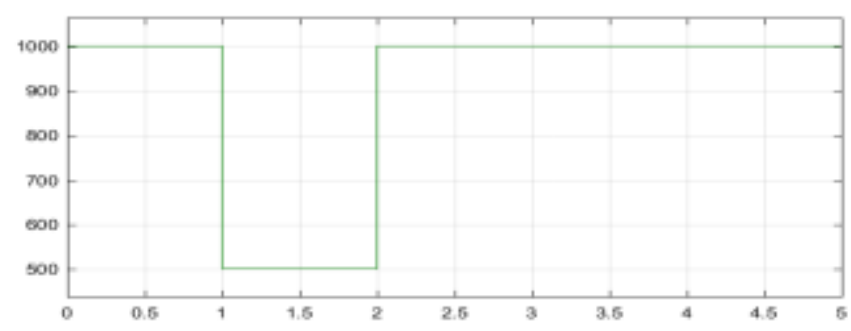

(b). Solar irradiance

Fig 12: Applied variation in grid voltage and irradiance

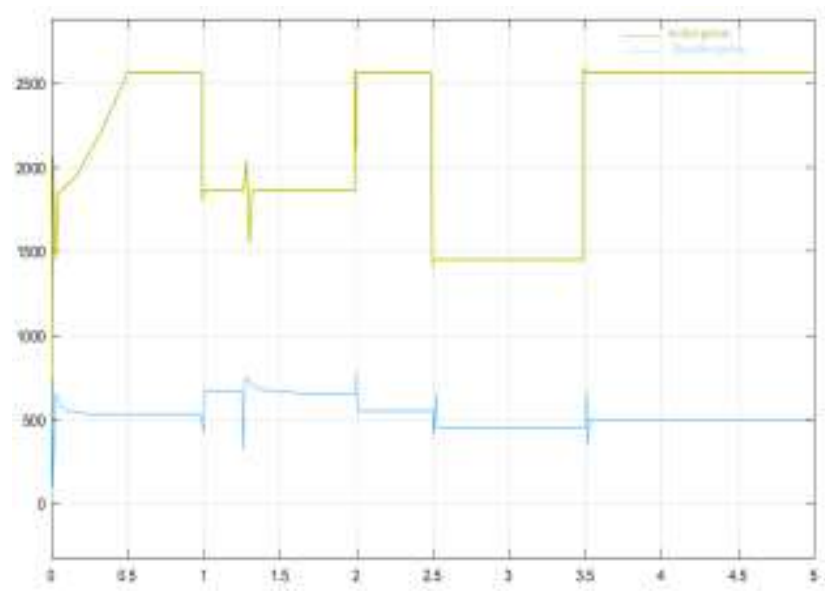

(a) Grid active and reactive power without using stability enhancing system

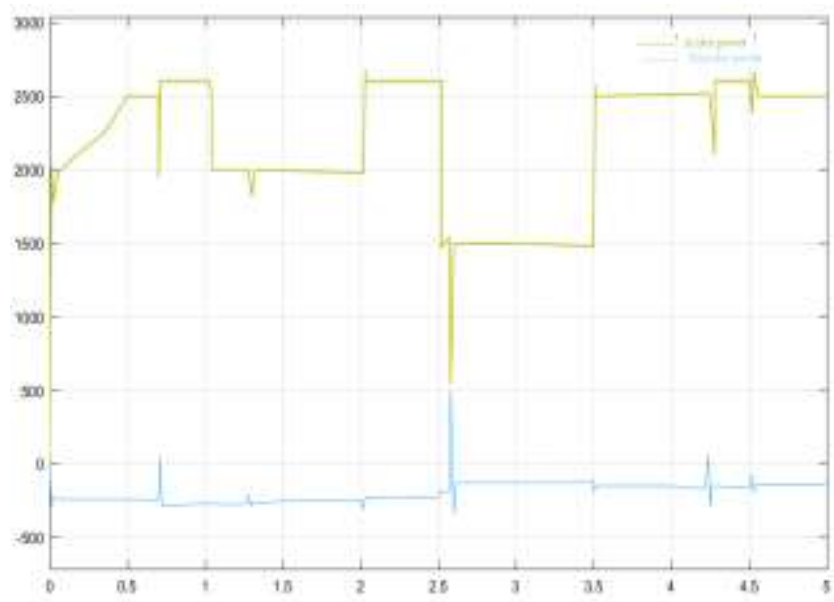

(b) Grid active and reactive power using stability enhancing system

Fig 13: Performance with and without stability enhancing system

\section{CONCLUSION}

Integration of battery with different controlling schemes in PV grid connected systems are studied. The optimization of battery will helps to reduce the costs and losses. The system with energy storage supports during the load dispatch and inverter control results in increased power quality and grid stability. Also during voltage fluctuations occurs in the grid, the system will provide the corresponding solution using energy storage. The power factor can also be improved with this stability enhancing system.

\section{ACKNOWLEDGEMENT}

We would like to thank the reviewers of this article for their insightful comments, which helped us to greatly improve its quality. The authors would like to thank Govt. Model Engineering college for the support during the study. 


\section{REFERENCES}

[1]. Yaosuo Xue, Liuchen Chang et.al.(2004), Topologies for single phase inverters for generator,overview IEEE Power Electronics,vol 10,No 5.

[2]. Ying huang, yu peng, meng huang, jianjun sun, xiaoming zha "co-ord ina tion control system for photovoltaic-based DC micro grid in proceedings with 2013,IEEE.

[3]. Xiong liu,peng wang, poh chiang loh " A hybrid AC/DC microgrid and its coordination control in proceedings with 2011 ,IEEE.

[4]. IEEE Standard for Interconnecting Distributed Resources with Electric Power Systems, IEEE Standard 1547-2003, Jul. 28, 2003.

[5]. L. H. Koh, Wang Peng, K. J. Tseng, Gao Zhi Yong, Reliability Evaluation of Electric Power Systems with Solar Photo vol taic \& Energy Storage, 2014 IEEE.

[6]. Kjaer SB, Pedersen JK, Blaabjerg F., "A review of single-phase grid-connected inverters for photovoltaic modules" IEEE Applied Electronics Sep./Oct. 2005;41(5):1292-306. 